\title{
Colaboración en ciencia-tecnología entre España/ Unión Europea y América Latina. Tendencias en biotecnología, ciencia de los alimentos y nanomateriales ${ }^{1}$
}

\author{
José Luis Molina², Miranda J. Lubbers, José Luis Briansó, I van Martínez (UAB) y \\ Alejandro A. Ruiz (UNAM)
}

\begin{abstract}
Resumen
A partir de un estudio de dos oleadas de las colaboraciones internacionales en patentes y artículos científicos entre instituciones de los países del Mercosur (más México y Chile) y países de la Unión Europea en los campos de biotecnología, ciencia de los alimentos y nanomateriales, ha sido posible identificar el rápido crecimiento de la colaboración en biotecnología, la emergencia del campo de nanomateriales en México y Brasil y el estancamiento de la colaboración en investigación en ciencias de los alimentos en ese período. México, Brasil y en menor medida Argentina, son los países líderes en esos campos, aunque Chile es un país activo en colaboraciones internacionales. A pesar que España es un importante actor en la estructura de colaboración, todavía no existe un patrón estable de colaboración entre Universidades e instituciones de investigación entre ambas partes.
\end{abstract}

Palabras clave: cienciometría, Triple Hélice, biotecnología, ciencia de los alimentos, nanomateriales, política científica, Unión Europea, Mercorsur.

\begin{abstract}
On the basis of a longitudinal study of international collaborations in patents and scientific articles between countries of Mercosur (plus Mexico and Chile) and countries from the European Union in the fields of biotechnology, food science and nanomaterials, it was possible to assess the fast growth of biotechnology, the emergence of nanomaterials in Mexico and Brazil and the stagnation of food science in the period. Mexico, Brazil and to a lesser degree Argentina are the leading countries, whereas Chile is relatively very active internationally as well. Although Spain is an important actor in the structure of collaboration, there is still not a stable institutional frame of collaboration among Universities and research institutes of both sides.
\end{abstract}

Key words: Sciencometrics, Triple Helix, Biotechnology, Food Science, Nanomaterials, Research Policies, European Union, Mercosur.

\footnotetext{
${ }^{1}$ Este artículo presenta los resultados del proyecto SEJ 2007-30289-E - Flujos de cooperación en cienciatecnología-industria España-América Latina para la concreción de una cartera de proyectos colaborativos, financiado a través del programa de Acciones Complementarias del MICINN, dirigido por el Dr. José Luis Briansó Pena.

2 Autor principal (joseluis.molina@uab.es). Los autores pertenecen a la Universitat Autònoma de Barcelona (UAB) excepto Alejandro A. Ruiz que pertenece a la UNAM de México.
} 


\section{I ntroducción}

En este artículo se presentan los resultados de la investigación realizada durante el año 2008 y el primer trimestre del 2009 orientada a diseñar un método sencillo para la identificación de tendencias en la colaboración científico-técnica entre los países de la Unión Europea y América Latina y poder así informar políticas en este campo. Para ello se han recogido información sobre patentes de la base de datos INPADOC por una parte (biotecnología y ciencia de los alimentos) y sobre artículos publicados en la Web of Science (WoS en lo sucesivo) por otra (biotecnología, ciencia de los alimentos y nanomateriales). En ambos casos se ha recogido información en dos períodos con el objetivo de identificar posibles tendencias. Estos períodos han sido los años 2003/04 y los años 2006/07.

Con el objetivo de focalizar la investigación, además de la selección de los campos científicos mencionados se han seleccionado de América Latina los países del Mercosur (Argentina, Brasil, Uruguay, Paraguay) más Chile y México (Mercosur+ en adelante) y sus relaciones con los países que componen la Unión Europea en la actualidad.

El análisis de esta información nos ha permitido identificar la estructura de colaboración existente entre la UE y Mercosur+ en los tres campos científicos, el lugar ocupado por cada país y posibles tendencias existentes. Entendemos que esta visión de conjunto permite identificar fácilmente qué instituciones y qué países son candidatos a la colaboración, dependiendo del campo científico, si se quiere incidir positivamente en su evolución futura. Igualmente, para el caso de España, es posible identificar el rol que ocupa en la estructura de colaboración en estos tres campos.

La investigación de patentes y publicaciones científicas, así como el análisis de las colaboraciones a nivel de autorías e instituciones, tiene su origen en la propuesta de "Triple Hélice" de Leydesdorff, L. \& Etzkowitz, H. (1996) según la cual los estados deben aplicar políticas de colaboración entre administraciones, empresasindustrias y universidades-centros de investigación científica, entendiendo que es en esta intersección en la que se desarrolla la innovación tecnologíca (González de la Fe, 2009). Así, las patentes por un lado y las colaboraciones en artículos científicos por otro, constituyen una primera aproximación a la medición de este espacio de intersección positiva. 
Presentaremos en primer lugar brevemente este concepto y a continuación presentaremos los resultados de patentes y la colaboración científica a través de artículos publicados en la WoS. Finalmente, presentaremos un resumen de los resultados alcanzados.

La estructura de la investigación y aplicaciones de la ciencia cambió de forma radical después de la II Guerra Mundial (Leydesdorf, 2006). La imagen de un científico trabajando en solitario y haciendo contribuciones relevantes al conocimiento fue rápidamente sustituida por grupos de investigación trabajando en el marco de instituciones y conectados entre sí a través de una amplia red de colaboraciones. Por otra parte, los diferentes gobiernos adquirieron un rol activo en la promoción de la investigación científica y su vinculación con organizaciones industriales públicas y privadas. Precisamente, este concepto pretende capturar la interdependencia de esos tres actores mencionados en el sistema de CienciaTecnología (gobiernos y regulaciones, Universidades-Centros de investigación y organizaciones productivas) al tiempo que constituye una propuesta de orientación de las políticas públicas.

El concepto de "Triple Hélice" captura el campo de las relaciones entre el Estado, Universidades y Empresas al tiempo que pretende orientar políticas públicas.

A pesar que el concepto de "Triple Hélice" puede funcionar bien dentro de los límites estatales (donde las políticas nacionales puede ser promulgadas y aplicadas) la emergencia de un creciente campo de colaboraciones internacionales es de especial interés debido al hecho que la existencia de regulaciones en este caso son limitadas o inexistentes. En este contexto, podemos hablar de un verdadero fenómeno "emergente" en el que la red de colaboraciones es en sí mismo el foco principal del análisis.

Las colaboraciones científicas internacionales pueden ser entendidas como un fenómeno emergente o de red, en tanto en cuanto están sujetas a pocas o nulas regulaciones.

Recientes investigaciones de este espacio internacional (Leydesdorf \& Wagner, en prensa) muestran cómo el enorme crecimiento de las colaboraciones internacionales en los últimos años presenta una estructura de centro-periferia, con un grupo central de países que, por tanto, están en posición de controlar el sistema en su conjunto. España ha pasado a formar parte recientemente de ese núcleo de países. 
En este sentido, Delgado-López-Cózar et al. (2009) muestran a partir de los datos de Essential Science Indicators de la WoS cómo España forma parte de ese núcleo ocupando la 9a posición a nivel internacional y la $5^{\underline{a}}$ en la Unión Europea (con el 11 y 6 o puestos respectivamente por lo que a citas se refiere), siendo Estados Unidos el líder solitario pero en un contexto de emergencia de nuevos actores clave en el sistema como son China, Corea del Sur, India y Brasil.

Por lo que se refiere a las patentes (las cuales podrían considerarse un buen indicador de colaboración entre investigación y empresa), se ha producido a partir del año 2000 el fenómeno de una reducción de las patentes promovidas por universidades en las economías avanzadas, tanto en términos relativos como absolutos (Leydesdorff \& Meyer, 2008). Esta reducción del interés de las universidades por las patentes puede ser explicada según los autores por el nuevo régimen de ranking universitario, el cual, por ahora, no contempla ni las patentes ni los spin-off alcanzados en el sistema de evaluación.

Por tanto, nuestra investigación cabe situarla en una coyuntura de crecimiento de las colaboraciones internacionales en publicaciones científicas por una parte y la reducción del número de patentes promovidas por Universidades por otra.

\section{Patentes UE-Mercosur+ en biotecnología y tecnología de los alimentos}

Para identificar las patentes conjuntas en biotecnología y ciencia de los alimentos entre los países de la Unión Europea y Mercosur+ se ha realizado una búsqueda en la base de datos especializada INPADOC de todas las familias de patentes en las que al menos un inventor/a o solicitante fuese de un país de la UE y de un país del Mercosur $+^{3}$.

En la Tabla 1 se muestran las patentes que cumplen esta condición para el campo de la biotecnología.

\footnotetext{
${ }^{3}$ Los códigos utilizados para familias de patentes relacionadas con la "biotecnología" a nivel de subclase, grupo o subgrupo son los siguientes: A01H001, A01H004, A61K038, A61K039, A61K048, C02F003/34, C40B010, C12N, C12P, C12Q, C12S, G01N027/327, G01N033/53, G01N033/54, G01N033/55, G01N033/57, H04N, H04Q, H04R, H04S, G01N033/68, G01N033/74, G01N033/76, G01N033/78, G01N033/88, G01N033/92, C40B040, C40B050, C40B070 y C40B080. Los códigos a nivel de clase para "agroalimentación" son los siguientes: A21, A22, A23.
} 


\begin{tabular}{|l|l|c|c|c|}
\hline \multirow{3}{*}{ País } & \multicolumn{2}{|l|}{$\begin{array}{l}\text { Número de familias de } \\
\text { patentes con al menos un } \\
\text { solicitante del país en } \\
\text { cuestión (todos los campos } \\
\text { científicos) }\end{array}$} & $\begin{array}{l}\text { Número de familias de patentes con al menos un } \\
\text { solicitante de cada grupo de países (UE-Mercosur+) en } \\
\text { biotecnología }\end{array}$ \\
\cline { 2 - 5 } & 18.596 & Total & $\begin{array}{c}\text { Desde } \\
\mathbf{2 0 0 3 / 0 4}\end{array}$ & $\begin{array}{c}\text { Desde } \\
\mathbf{2 0 0 6 / 0 7}\end{array}$ \\
\hline Argentina & 140.684 & 81 & 6 & 28 \\
Brasil & 53 & 135 & 33 & 15 \\
Paraguay & 1.056 & 3 & 0 & 0 \\
Uruguay & 1.031 & 16 & 3 & 8 \\
\hline Chile & 21 & 1 & 2 \\
\hline
\end{tabular}

Tabla 1. Número total de patentes en biotecnología durante el período 2003/04 y el período 2006/07 con miembros de al menos un país de cada grupo (UE-Mercosur+, excepto México en este caso).

Destaca en primer lugar la escasísima importancia de las patentes del espacio UEMercosur+ (menos México en este caso) en relación al número total de patentes inscritas por cada país de las mismas familias, si bien es cierto que la primera columna contabiliza las patentes en los períodos en cuestión en todos los campos científicos. Igualmente, aunque poco relevante en términos absolutos, destaca el rápido crecimiento de las patentes con países de la UE de Argentina.

En el caso de la tecnología de los alimentos la situación es todavía más acentuada: no solamente el número total de patentes internacionales con países de la UE es muy pequeño sino que se observa una situación de estancamiento en todos los casos.

\begin{tabular}{|l|c|c|c|c|}
\hline \multirow{2}{*}{ País } & $\begin{array}{c}\text { Número de familias de } \\
\text { patentes con al menos } \\
\text { un solicitante del país en } \\
\text { cuestión (todos los } \\
\text { campos científicos) }\end{array}$ & $\begin{array}{l}\text { Número de familias de patentes con al menos un } \\
\text { solicitante de cada grupo de países (UE-Mercosur+) en } \\
\text { tecnología de los alimentos }\end{array}$ \\
\cline { 2 - 5 } & 18.596 & Total & Desde 2003/ '04 & Desde 2006/ '07 \\
\hline Argentina & 140.684 & 28 & 4 & 4 \\
Brasil & 53 & 62 & 7 & 6 \\
Paraguay & 1.056 & 2 & 2 & 1 \\
Uruguay & 1.031 & 13 & 2 & 2 \\
\hline Chile & & & & 1 \\
\hline
\end{tabular}

Tabla 2. Número total de patentes en ciencia de los alimentos durante el período 2003/04 y el período 2006/07 con miembros de al menos un país de cada grupo (UE-Mercosur+, excepto México en este caso).

La información sobre patentes no es pública por lo que es difícil y costoso poder hacer un análisis en profundidad. Sin embargo esta tendencia mostrada de un relativo dinamismo del campo de la biotecnología y un estancamiento del campo de la tecnología de los alimentos (por lo que al espacio de colaboración UE-Mercosur+ se refiere) ha podido ser contrastada con los datos procedentes de los artículos científicos publicados en revistas indexadas en la WoS. Pasemos a examinar esta cuestión. |ADV 
El relativo dinamismo en el campo de las patentes en biotecnología contrasta con el estancamiento de las colaboraciones de la UE-Mercosur+ en tecnología de los alimentos

\section{Artículos publicados en la WoS en biotecnología, ciencia de los alimentos y nanomateriales}

La búsqueda en la Web de la Ciencia (el servicio central de la WoS) incluyó las siguientes bases de datos: el Science Citation Index ( $\mathrm{SCl}$ )-Expanded, el Social Science Citation Index (SSCI) y también el Arts and Humanities Citation Index $(\mathrm{A} \& \mathrm{HCl})$. Para cada campo científico se realizaron búsquedas separadas (biotecnología, ciencia de los alimentos y nanomateriales) en dos fases en cada caso, una para el período 2003-2004 y la segunda para el período 2006-2007. En la búsqueda la palabra clave fue el campo en cuestión, indicada el campo TS, lo cual significa que la palabra clave puede aparecer en el título del artículo, el resumen, las palabras clave proporcionadas por los autores o bien por la misma WoS. Como en el caso anterior al menos un autor debe pertener a cada bloque de países para poder ser seleccionado. El resultado fue de 403 artículos en total, teniendo en cuenta los tres campos científicos y los dos períodos de búsqueda.

Hemos seguido este criterio "fuerte" de selección de artículos en lugar de realizar búsquedas por subcriterios, más sofisticadas y con más posibilidades de encontrar documentos relacionados. Aunque en algunos casos los subcriterios elegidos pueden estar basados en investigaciones previas (Cf. Kostoff 2007 y Porter et al. 2008 para el caso de nanotecnología, con un 30\% de documentos no coincidentes entre ambos métodos), el interés comparativo de este estudio aconsejaba el empleo de un criterio sencillo y unívoco que pudiese replicarse fácilmente. Esto explica que el número de documentos estudiado sea muy inferior al que se puede obtener mediante otros criterios de búsqueda, pero entendemos que esta reducción queda justificada por la claridad del criterio utilizado para la comparación de los dos períodos seleccionados y los diferentes campos de conocimiento.

La Tabla 3 indica los artículos por campo y período. A efectos comparativos se añade la producción española en los mismos campos y períodos. Esta comparación permite ver fácilmente como los países del Mercosur+ han publicado relativamente pocos artículos en revistas indexadas en la WoS en estos campos. I gualmente, es destacable observar las mismas tendencias en los tres campos en los dos casos (indicada con una flecha ascendente $\uparrow$ o descendente $\downarrow$ según los casos). 


\begin{tabular}{|l|c|c|c|c|}
\hline \multirow{2}{*}{ Campo } & \multicolumn{2}{|c|}{ Mercosur+ } & \multicolumn{2}{c|}{ España } \\
\cline { 2 - 5 } & $\mathbf{2 0 0 3 - 2 0 0 4}$ & $\mathbf{2 0 0 6 - 2 0 0 7}$ & $\mathbf{2 0 0 3 - 2 0 0 4}$ & $\mathbf{2 0 0 6 - 2 0 0 7}$ \\
\hline Biotecnología & 59 & $87 \uparrow$ & 47 & $78 \uparrow$ \\
Ciencia de los Alimentos & 145 & $69 \downarrow$ & 124 & $46 \downarrow$ \\
Nanomateriales & 14 & $32 \uparrow$ & 17 & 157 \\
\hline Total (sin solapamientos & 217 & 186 & & \\
entre campos) & \multicolumn{2}{|c|}{$\mathbf{4 0 3}$} & \multicolumn{2}{c|}{$\mathbf{3 4 5}$} \\
\hline Total artículos WoS & \multicolumn{2}{|c|}{} & & \\
\hline
\end{tabular}

Tabla 3. Número de artículos publicados en los periodos en 2003/04 y 2006/07 en los campos de biotecnología, ciencia de los alimentos y nanomateriales con al menos un autor/a de cada uno de los dos bloques de países (UE-Mercosur+). España se presenta como ejemplo comparativo.

En base de esta misma producción científica hemos medido el número de artículos publicados tanto a nivel de institución como de país, así como el número de copublicaciones entre instituciones. Para identificar los actores más importantes del Mercosur+, hemos considerado, entre otros: (1) el número de artículos publicados por período, (2) el número de copublicaciones con otros instituciones (centralidad de grado), (3) el número de copublicaciones con instituciones internacionales y (4) la medida en que una institución forma una "puente" entre otras instituciones (centralidad de intermediación). Para ello, hemos calculado las frecuencias de publicaciones (1) con el software SPSS, y la centralidad de grado (2), el número de colaboraciones internacionales (3) y la centralidad de intermediación (4) con el software UCINET (Borgatti et al., 2002). Para analizar la estabilidad temporal de los rankings, hemos calculado en SPSS el coeficiente de correlación de Pearson entre los rankings de los dos períodos, basándonos en el conjunto de instituciones que han publicados artículos en el WoS en ambos períodos. Por último, se han elaborado visualizaciones de las colaboraciones con el software Pajek (Batagelj \& Mrvar, 2003), que permiten una comprensión rápida de la estructura general de las colaboraciones y la identificación de las actores más importantes en este campo. En las visualizaciones, los nodos representan países (en la Figura 1) o instituciones (en las demás figuras) y las líneas indican las copublicaciones entre ellas. Cabe mencionar que una colaboración entre tres o más instituciones se representa por un conglomerado completamente conectado de tres o más nodos. Para valorar el desarrollo de las colaboraciones en el tiempo, se pueden comparar los grafos de las dos oleadas. Hay que mencionar que hemos optado por no fijar las posiciones de los actores en el grafo en el tiempo, ya que eso tiende a ocultar mucho la estructura de conglomerados.

Observemos en primer lugar a los actores en la producción científica anteriormente identificada. Las publicaciones mencionadas en la Tabla 3 han sido producidas en el caso de Mercosur+ por 131 instituciones (76 brasileñas, 49 mexicanas, 40 argentinas, 21 chilenas y 6 de Uruguay) pero el nivel de producción varía 
notablemente. En la Tabla 4 se presentan las 11 instituciones más productivas, las cuales producen la mitad de los artículos identificados.

\begin{tabular}{|l|l|c|}
\hline N & Institución & N artículos \\
& & \\
\hline 1 & Universidade de Sao Paulo (Brasil) & 36 \\
2 & Universidad Nacional Autónoma de México (México) & 33 \\
3 & Universidade Estadual de Campinas (Brasil) & 27 \\
4 & Universidad de Buenos Aires (Argentina) & 23 \\
5 & Consejo Nacional de Investigaciones Científicas y Técnicas (Argentina) & 17 \\
\hline 6 & Universidade Federal do Rio de Janeiro (Brasil) & 17 \\
7 & Universidade Estadual Paulista Julio de Mesquita Filho (Brazil) & 16 \\
8 & Pontificia Universidad Católica de Chile (Chile) & 13 \\
9 & Instituto Politécnico Nacional (México) & 12 \\
10 & Empresa Brasileira de Pesquisa Agropecuaria (Brasil) & 11 \\
\hline 11 & Universidad Nacional de Mar del Plata (Argentina) & 11 \\
\hline
\end{tabular}

Tabla 4. Listado de instituciones del Mercosur+ con más de 10 artículos publicados en los campos de biotecnología, ciencia de los alimentos y nanomateriales en los dos períodos estudiados (2003/04 y 2006/07).

En los siguientes apartados tratamos las colaboraciones entre instituciones en los diferentes campos, empezando con un resumen a nivel de país.

\section{Colaboración UE-Mercosur+ a nivel de país}

Para obtener una impresión general de la colaboración en estos campos al nivel de país se ha elaborado la Figura 1. En ella los nodos representan países (con su etiqueta) y la relación entre dos nodos existe si hay al menos un artículo conjunto entre investigadores/as de ambos países. Podemos ver cómo México, Brasil, Argentina y Chile son cuatro centros importantes de colaboración y cómo algunos países como Estados Unidos, por ejemplo, ocupan una posición central (esto es, mantienen colaboraciones con todos o gran parte de los países).

Hay que tener en cuenta que solamente se recogen las colaboraciones que incluyen algún país del Mercosur+, por lo que las colaboraciones existentes independientes de estos países no son tenidas en cuenta en este caso. 


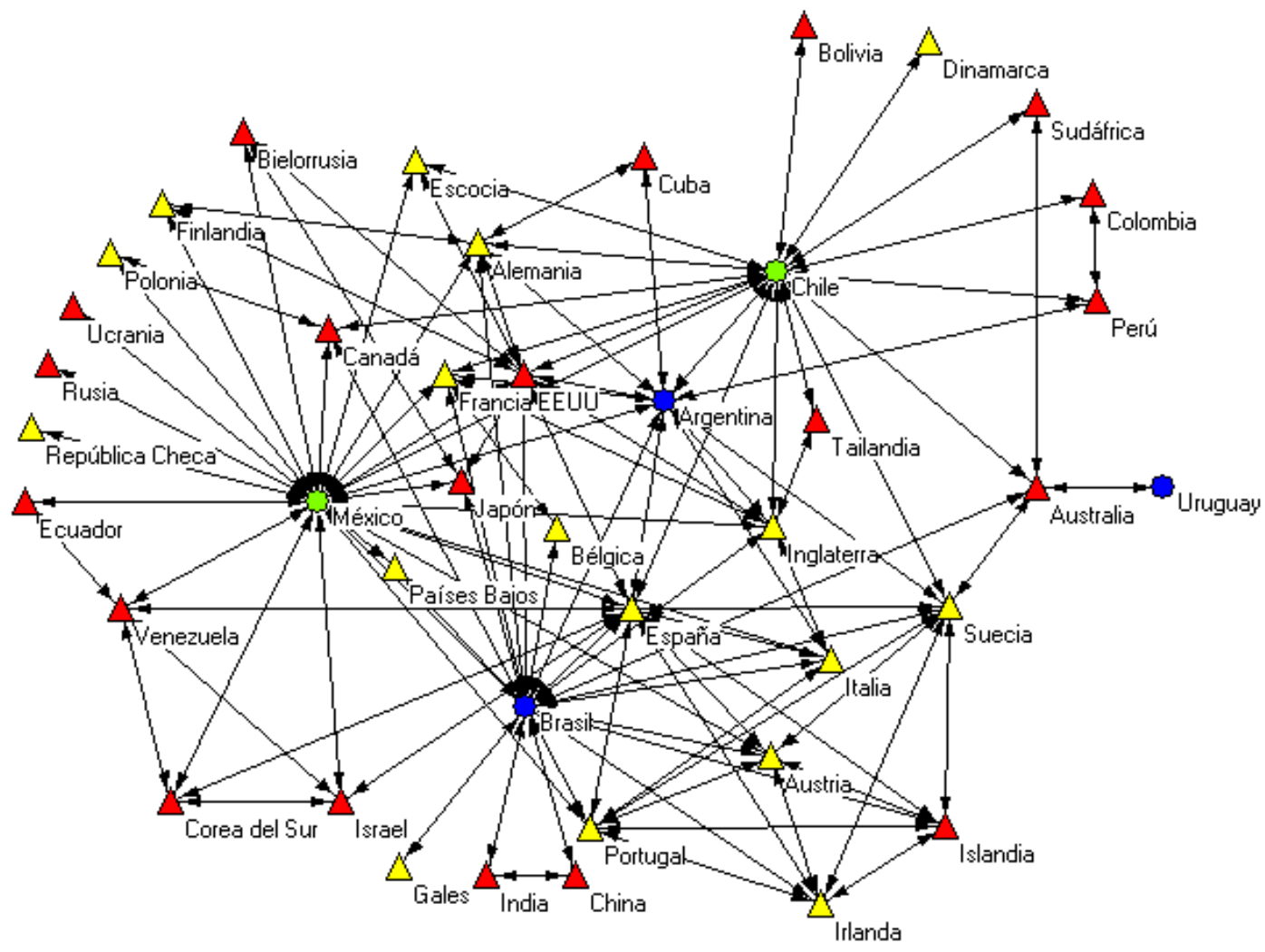

Figura 1. Colaboraciones entre los países del Mercosur+ y otros países. Los nodos azules redondos indican países del Mercosur, los nodos verdes hacen referencia a México y Chile, los triángulos amarillos indican países de la UE y los triángulos rojos el resto de países.

Por lo que se refiere a las colaboraciones con países de la UE, en la Tabla 5 encontramos colaboraciones con 15 países, si bien solamente Alemania, El Reino Unido y España mantienen más de diez colaboraciones con Argentina, Brasil, Chile y México.

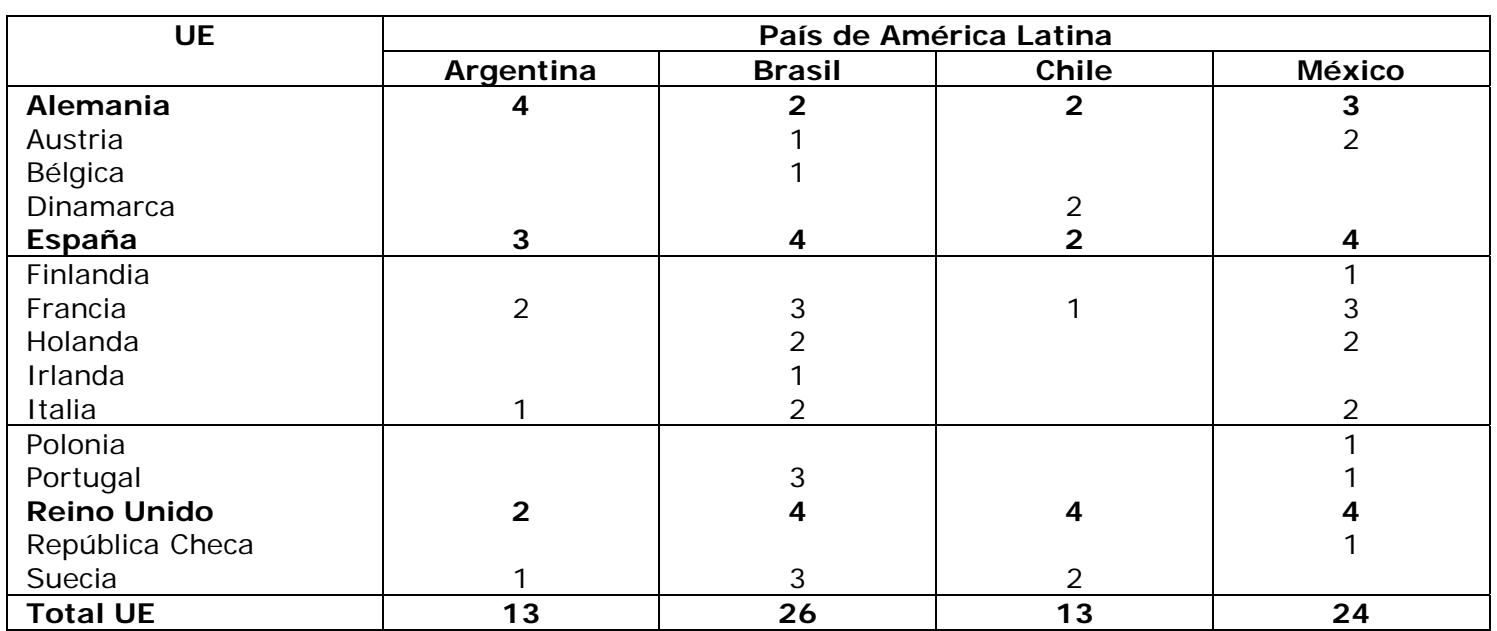

Tabla 5. Colaboraciones en los tres campos y en los dos períodos entre los dos bloques de países (UEMercosur+). 
A continuación presentaremos una serie de dos grafos, uno por período, para cada uno de los campos estudiados. De esta forma es posible apreciar la evolución de la estructura de la colaboración entre los dos bloques de países.

\section{Evolución de la colaboración UE-Mercosur+ en biotecnología}

En biotecnología Brasil es el país que tiene más publicaciones conjuntas ( 67 en total) y, además, presenta un rápido crecimiento si se comparan los dos períodos (ver Tabla 6). También es posible ver un número creciente de publicaciones conjuntas en el caso de México (39), mantenido Argentina y Chile una apreciable actividad.

\begin{tabular}{|l|c|c|c|}
\hline \multirow{2}{*}{ País } & \multicolumn{2}{|c|}{ Número total de artículos conjuntos en biotecnología } \\
\cline { 2 - 4 } & $\mathbf{2 0 0 3 / 0 4}$ & $\mathbf{2 0 0 6 / 0 7}$ & Total \\
\hline Argentina & 12 & 12 & 24 \\
Brasil & 23 & $44 \uparrow$ & 67 \\
Uruguay & 3 & 0 & 3 \\
\hline Chile & 7 & 8 & 15 \\
México & 14 & $25 \uparrow$ & 39 \\
\hline
\end{tabular}

Tabla 6. Número total de artículos en biotecnología con países del Mercosur+.

En biotecnología Brasil es el actor principal y más dinámico del campo de colaboración UE-Mercosur+.

Además, es posible apreciar cómo el volumen de colaboración interinstitucional en biotecnología ha crecido a lo largo del tiempo. En el período 2003/04 los institutos del Mercosur+ que habían publicado artículos en biotecnología colaboraban con una media de 1,02 institutos por artículo $(S D=0,87)$ mientras que en el período 2006/07 había aumentado a 1,77 (SD=1,61). Veintitrés instituciones del Mercosur+ aparecen en las dos oleadas; para ellos, la correlación entre el número de colaboraciones que tenían en el 2003/04 y en el 2006/07 es de 0,39 ( $p=0,07)$.

El dinamismo de la estructura de colaboración en el campo de la biotecnología se puede apreciar comparando las Figuras 2 y 3 . En esas figuras los nodos representan las universidades o institutos, el tamaño representan el número de artículos publicados y las líneas, las co-publicaciones y su grosor indica el volumen de colaboración. Así, cuanta más gruesa sea la línea, más copublicaciones había entre las instituciones.

La comparación de las dos figuras muestra cómo la estructura de colaboración adquiere complejidad con el tiempo. Así, mientras en el período 2003/04 los artículos eran producidos por una sola institución en solitario o con pocas colaboraciones, en el 2006/07 podemos observar la existencia de más actores y 
más relacionados entre sí. Los actores más importantes son la Universidade Federal do Rio de Janeiro (etiquetada con un "6"), la Universidade de Sao Paulo ("1") y la UNAM ("2"). En el caso de España encontramos el Institut de Recerca i Tecnologia Agroalimentaries ("292"), la Universidad de Córdoba (“53"), el CSIC ("136"), el CIFA Alameda del Obispo IFAPA ("126") y la Universidad de Santiago de Compostela ("48"), la única institución española que aparece en el período anterior.
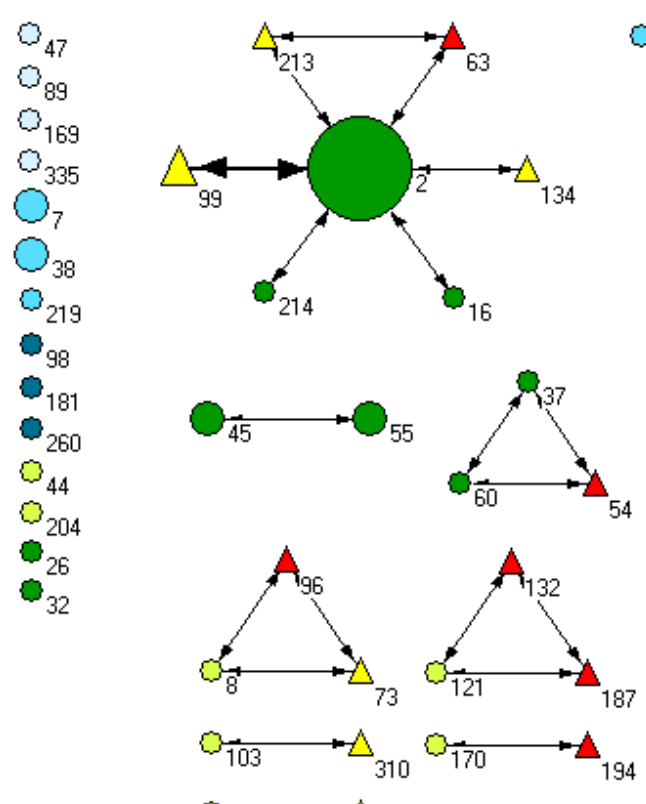

$0 \stackrel{-\triangle}{333}$
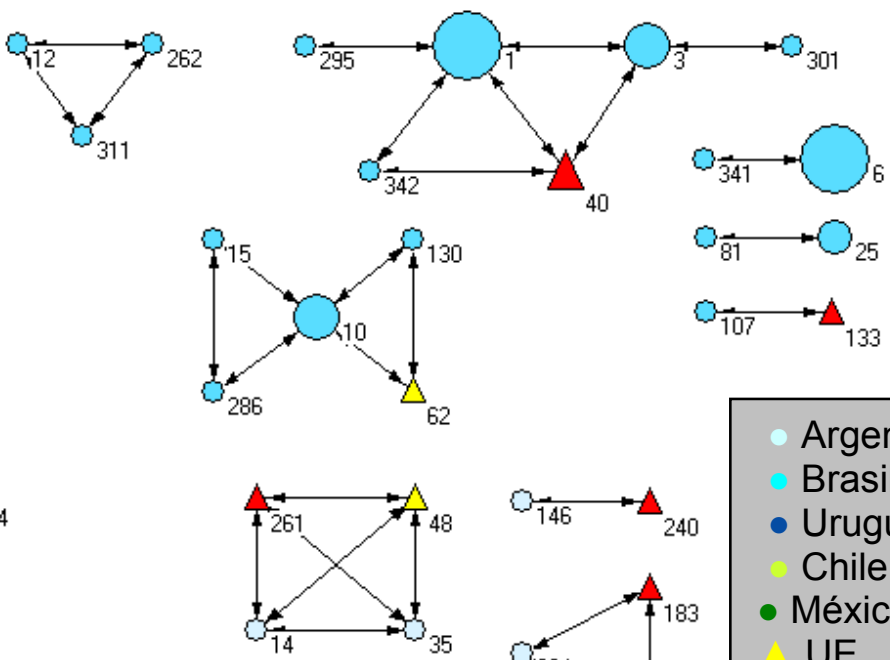

Argentina

Brasil

- Uruguay

Chile

- México

UE

$\triangle$ Otros

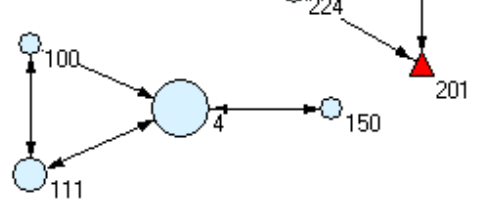

Figura 2. Colaboraciones en biotecnología 2003/04.

Nota: Los números indican instituciones (las 11 más importantes aparecen numeradas en la tabla 4, los demás números se pueden consultar en el informe disponible en el informe final del proyecto en www.egoredes.net). El tamaño de los nodos representa el número de artículos escritos por la institución y el grosor de la flecha el número de colaboraciones. 


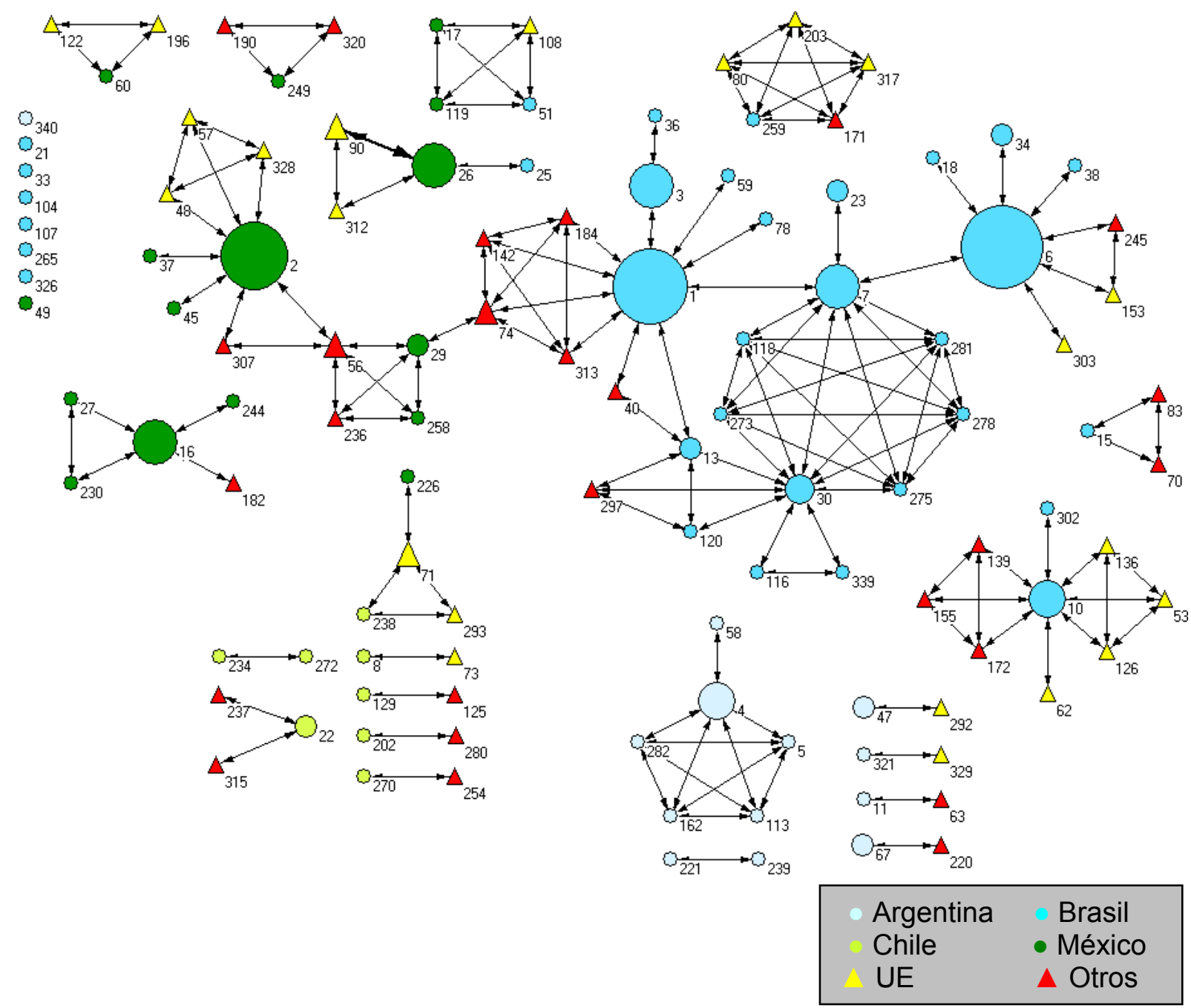

Figura 3. Colaboraciones en biotecnología 2006/07.

\section{Evolución de la colaboración UE-Mercosur+ en ciencia de los alimentos}

A pesar que el campo de ciencia de los alimentos presenta el mayor número de publicaciones, por lo que se refiere tanto a la estructura de colaboración como a la evolución temporal, encontramos no solamente una reducción de su complejidad sino un estancamiento de la producción. De nuevo Brasil con 92 publicaciones lidera el área, seguido de Argentina (56) y México (44), ver Tabla 7.

\begin{tabular}{|l|c|c|c|}
\hline \multirow{2}{*}{ País } & \multicolumn{3}{|c|}{ Número total de artículos conjuntos en ciencia de los alimentos } \\
\cline { 2 - 4 } & $\mathbf{2 0 0 3 / 0 4}$ & $\mathbf{2 0 0 6 / 0 7}$ & Total \\
\hline Argentina & 39 & $17 \downarrow$ & 56 \\
Brasil & 60 & $32 \downarrow$ & 92 \\
Uruguay & 2 & $1 \downarrow$ & 3 \\
\hline Chile & 16 & $9 \downarrow$ & 25 \\
México & 31 & $13 \downarrow$ & 44 \\
\hline
\end{tabular}

Tabla 7. Número total de artículos en ciencia de los alimentos con países del Mercosur+. 
En el período 2003/04 los institutos del Mercosur+ que habían publicado artículos en la ciencia de los alimentos colaboraban con una media de 1,21 institutos por artículo (SD $=1,01$ ) mientras que en el período 2006/07 había aumentado ligeramente a $1,39(S D=1,20)$.

En las figuras 4 y 5 se ilustra la evolución observada. En el período 2003/04 los principales actores de Mercosur+ son la Universidade de Sao Paulo ("1"), Universidade Estadual de Campinas ("3"), Universidad de Buenos Aires ("4"), Consejo Nacional de Investigaciones Científicas y Técnicas de Argentina ("5") y la Universidade Estadual Paulista Julio de Mesquita Filho ("7"). La Universidade de Sao Paulo, aparte de ser uno de los actores más productivos también dispone de una posición muy central en la red, ejerciciendo un papel de conexión de diferentes partes del sistema. La Universidade Federal de Sao Paulo ("28") por su parte, a pesar de tener un volumen de publicaciones inferior, también ejerce este papel de "puente". Cuatro universidades españolas aparecen en las colaboraciones durante 2003/04: la Universidad de Cordoba ("53"), la Universidad Politécnica de Valencia ("222"), la Universitat Politécnica de Catalunya (“304”) y la Universidad de Jaén (“250").

En cambio, en el período 2006/07 (figura 5), podemos apreciar una estructura mucho más sencilla y con menos colaboraciones con países de la UE, con la excepción de un artículo que fue el resultado de una colaboración entre 11 institutos. Dos universidades españolas aparecen en las colaboraciones: la Universitat de Lleida ("322") y la Universidad de Santiago de Compostela ("48").

Treinta y cuatro instituciones del Mercosur+ aparecen en las dos oleadas; para ellas la correlación entre el número de colaboraciones que tenían en el 2003/04 y en el $2006 / 07$ es de $-0,07(p=0,68)$. Esto indica que el ranking observado en la primera oleada no tiene estabilidad temporal. 


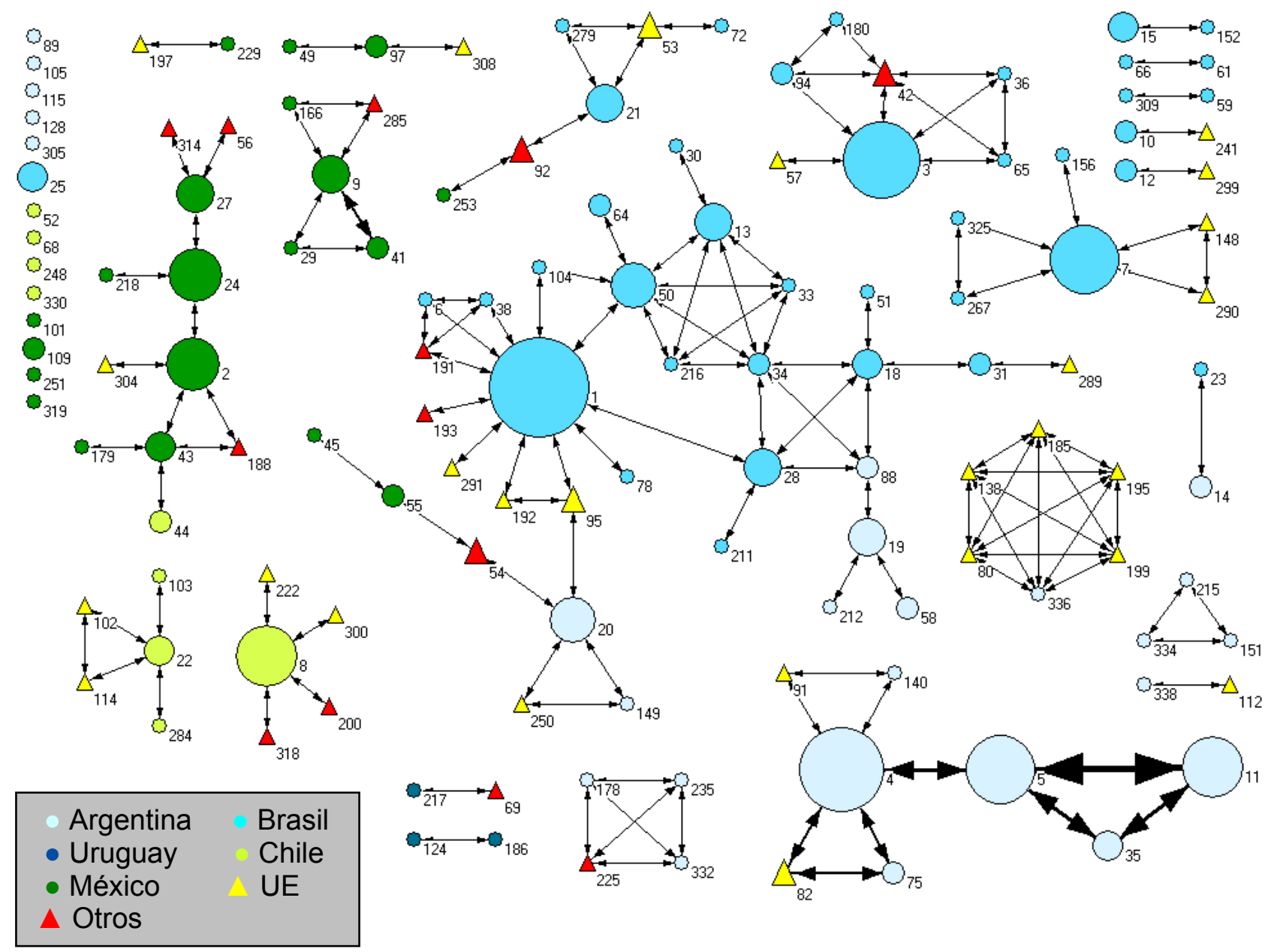

Figura 4. Colaboraciones en ciencia de los alimentos 2003/04. 


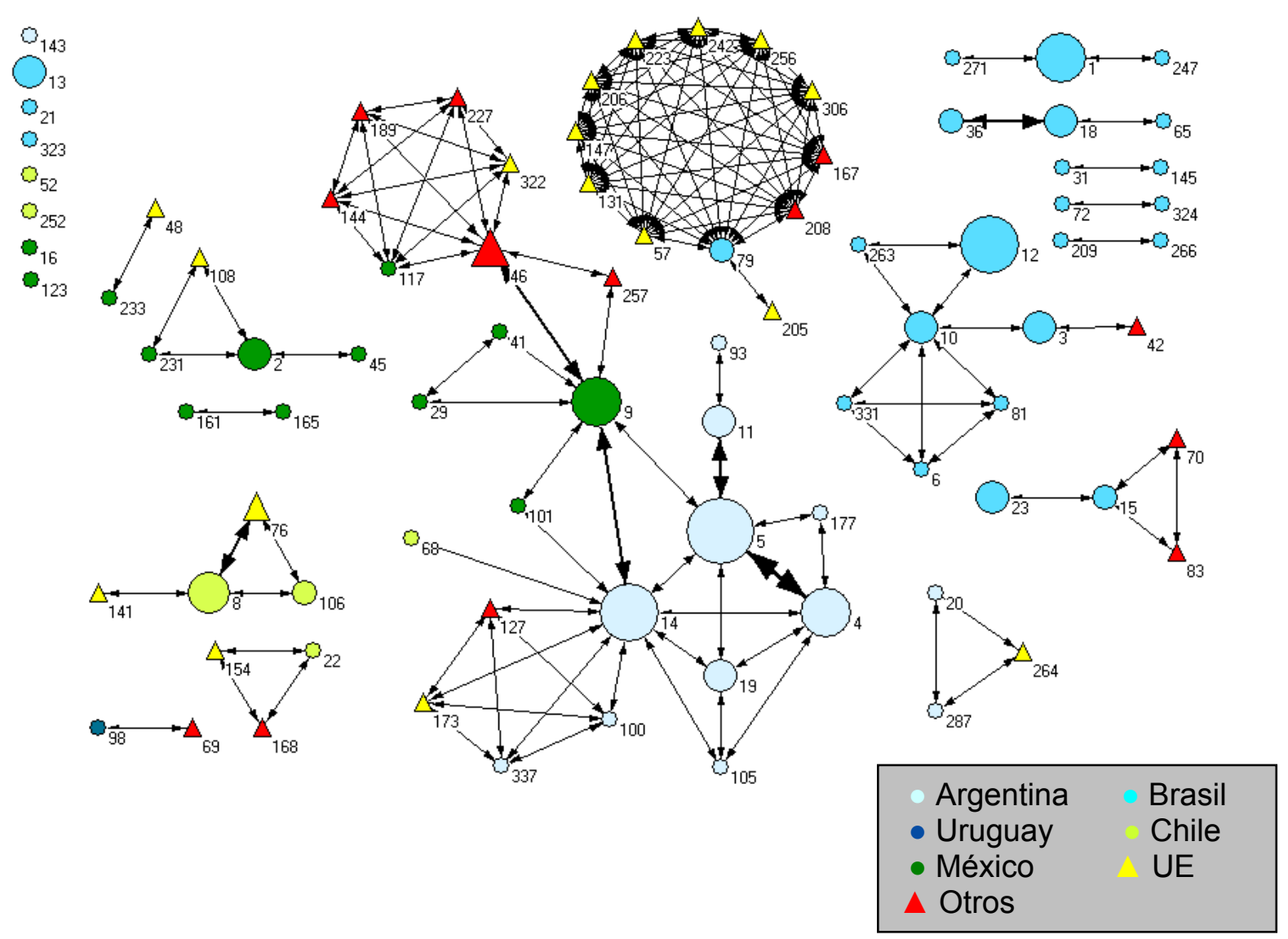

Figura 5. Colaboraciones en ciencia de los alimentos 2006/07.

Es necesaria realizar una nueva oleada para poder determinar si esta tendencia es conyuntural o si por el contrario se consolida.

Con esto pasamos al último campo investigado: los nanomateriales.

\section{Evolución de la colaboración UE-Mercosur+ en nanomateriales}

En el campo de los nanomateriales México es el actor más importante con 23 publicaciones, seguido de Brasil con 18, ver Tabla 8. En general, podemos observar que el número de artículos en nanomateriales se ha incrementado en el tiempo, al igual que las colaboraciones inter-institucionales, pasando de una media de 1.02 colaboraciones por artículo en el 2003/04 (SD $=0,81$ ) a 1,46 por artículo en 2006/07 (SD $=0,87)$.

\begin{tabular}{|l|c|c|c|}
\hline \multirow{2}{*}{ País } & \multicolumn{2}{|c|}{ Número total de artículos conjuntos en nanomateriales } \\
\cline { 2 - 4 } & $\mathbf{2 0 0 3 / 0 4}$ & $\mathbf{2 0 0 6 / 0 7}$ & Total \\
\hline Argentina & 3 & 1 & 4 \\
Brasil & 3 & $15 \uparrow$ & 18 \\
Paraguay & 0 & 0 & 0 \\
Uruguay & 0 & 0 & 0 \\
\hline Chile & 1 & 0 & 1 \\
México & 7 & $16 \uparrow$ & 23 \\
\hline
\end{tabular}

Tabla 8. Número total de artículos en nanomateriales con países del Mercosur+. 
Aunque la producción absoluta es reducida puede observarse en este campo una tendencia de crecimiento (ver Figuras 6 y 7). La estructura de colaboración fue algo más compleja en el segundo período, siendo los actores principales la UNAM de nuevo ("2"), la Universidade de Sao Paulo ("1"), la Universidade Estadual de Campinas ("3") y el Instituto Potosino de Investigación Científica y Tecnológica ("17"). También puede observarse un ligero aumento de la colaboración UEMercosur+, pero no se encontraron colaboraciones con institutos españoles en ninguno de los dos períodos.

Sólo 8 instituciones del Mercosur+ aparecen en las dos oleadas. En este caso la correlación entre el número de colaboraciones mantenidas en el 2003/04 y en el $2006 / 07$ es de $0,45(p=0,26)$.
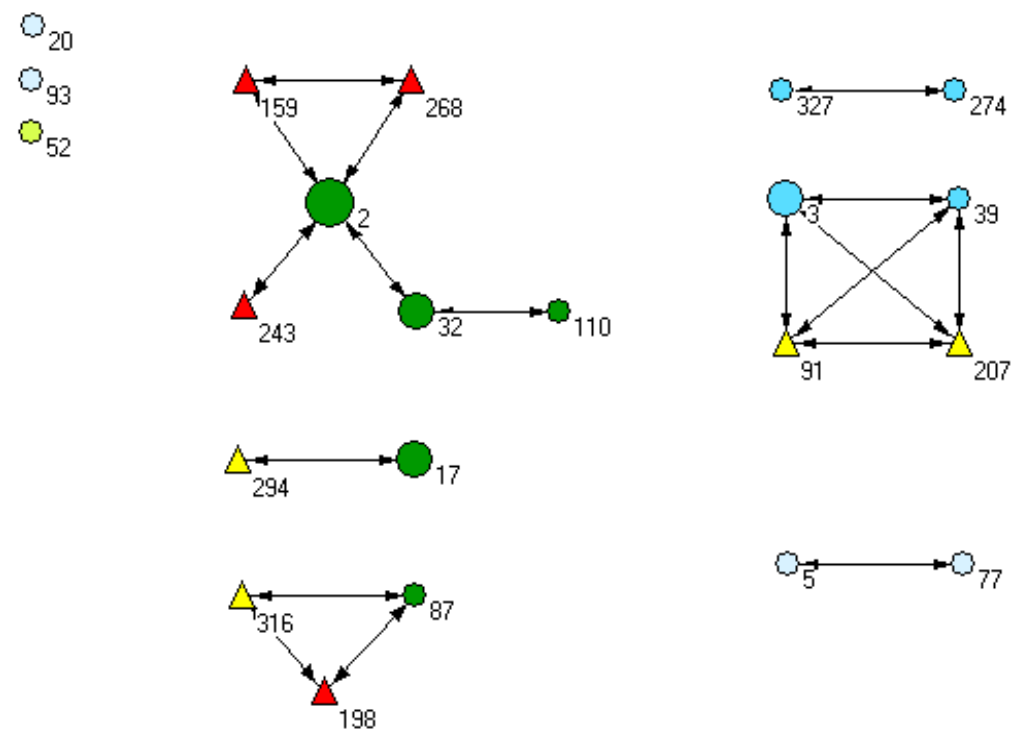

Argentina Brasil Chile

- México

UE

Otros

Figura 6. Colaboraciones en nanomateriales 2003/04. 

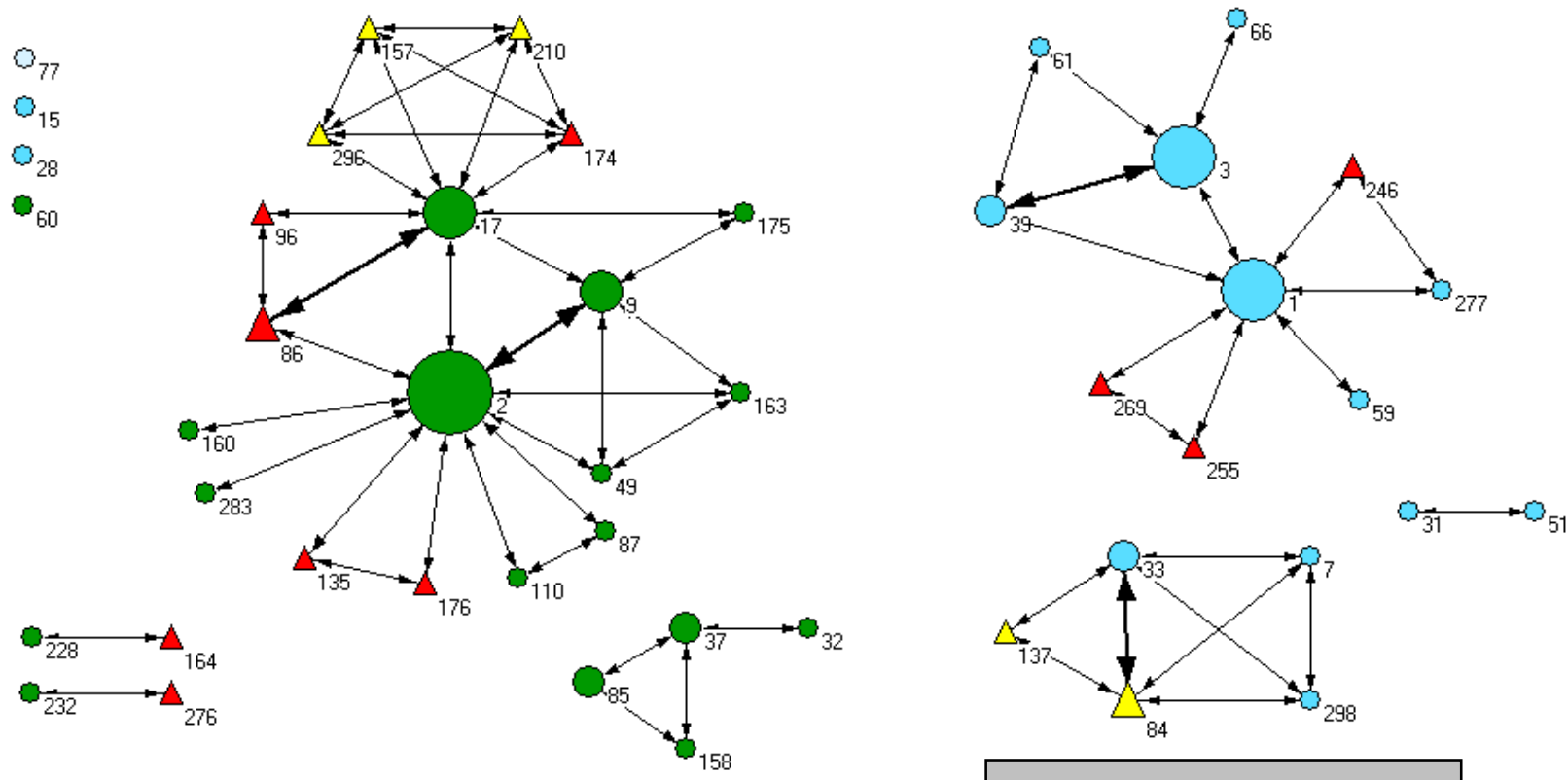

\begin{tabular}{|ll|}
\hline Argentina & Brasil \\
México & UE \\
$\Delta$ Otros & \\
\hline
\end{tabular}

Figura 7. Colaboraciones en nanomateriales 2006/07.

\section{Conclusiones}

El espacio de colaboración UE-Mercosur+ en los campos de la biotecnología, ciencia de los alimentos y nanomateriales es una realidad, si bien con diferente importancia y grado de dinamismo. Así, ha sido posible apreciar un gran dinamismo en el campo de la colaboración en biotecnología, un estancamiento en el campo de la ciencia de los alimentos (a pesar de partir de niveles de producción superiores) y la emergencia del campo de los nanomateriales, sobre todo en México y Brasil.

Solamente 10 instituciones del espacio Mercosur+ son responsables de la mitad de la producción agregadas en estos tres campos. Brasil, México, Argentina y Chile son, por este orden, los principales actores en el sistema.

Entendemos que gracias al enfoque longitudinal adoptado y a su representación gráfica, es posible identificar tendencias de la colaboración en campos científicos seleccionados tanto por lo que se refiere a la producción absoluta como a la misma estructura de colaboración. I gualmente, es posible identificar países y actores clave y anticipar, así, los resultados de posibles iniciativas. En el caso de España es interesante constatar que España es uno de los países de la UE que tiene más colaboraciones con países del MERCOSUR, pero aún así, la cantidad de colaboraciones es reducida, lo cual sugiere un campo de desarrollo todavía por explotar. 
Por último, la comparación de las tendencias observadas en patentes y artículos científicos nos permite disponer de indicadores independientes del sistema de ciencia y tecnología y, por tanto, interpretar mejor los resultados alcanzados.

\section{Bibliografía}

Barrere, R. et al. (2008). "La nanotecnología en I beroamérica: situación actual y tendencias" en El Estado de la Ciencia 2008, Observatorio Iberoamericano de la Ciencia, Tecnología e Innovación del Centro de Altos Estudios Universitarios de la Organización de Estados Iberoamericanos (OEI) <www.oei.es>.

Batagelj, V., \& A. Mrvar (2003). Pajek - Analysis and visualisation of large networks. In M. Jünger \& P. Mutzel (Eds), Graph Drawing Software (77-103). Berlin, Germany: Springer (Series Mathematics and Visualization).

Borgatti, S. P., M. G. Everett, \& L. C. Freeman (2002). UCINET for Windows: Software for Social Network Analysis. Harvard, MA: Analytic Technologies.

Delgado-López-Cózar, E., E. Jiménez-Contreras \& R. Ruiz-Pérez "España y los 25 grandes de la ciencia mundial en cifras (1992-2008)", El profesional de la información, 2009, vol.18, n. 1, enero-febrero.

Fernández, MT; I. Gómez, \& J. Sebastian (1998). “La cooperación científica de los países de América Latina a través de estudios bibliométricos", Interciencia 23, 6 (328-337).

González de la Fe, T. (2009). "El modelo de Triple Hélice de relaciones universidad, industria y gobierno: un análisis crítico", ARBOR - Ciencia, Pensamiento y Cultura, CLXXXV 738 julio-agosto (739-755).

Kostoff, R. N., R. G. Koytcheff, \& C. G. Y. Lau (2007). "Global nanotechnology research literature overview", Technological Forecasting and Social Change, Volume 74, Issue 9, 1733-1747, DOI: 10.1016/j.techfore.2007.04.004.

Leydesdorff, L. \& H. Etzkowitz (1996). “Emergence of a Triple Helix of UniversityIndustry-Government Relations", Science and Public Policy, vol. 23, 5 (279-286).

Leydesdorff, L. (2006). The Knowledge-based Economy. Modeled, measured, simulated. Boca-Ratón, FL: Universal Publishers.

Leydesdorff, L. \& M. Meyer (en prensa). The Decline of University Patenting and the End of the Bayh-Dole Effect. http://users.fmg.uva.nl/lleydesdorff/BayhDole/index.htm.

Leydesdorff, L., \& C. S. Wagner (en prensa). "International Collaboration in Science and the Formation of a Core Group", Journal of Informetrics. http://users.fmg.uva.nl/lleydesdorff/cswagner07/index.htm.

Porter, A. L., J. Youtie, P. Shapira, \& D. J. Schoeneck (2008), "Refining search terms for nanotechnology", J ournal of Nanoparticle Research, vol. 10, 715-728. 
Wagner, C. S., \& L. Leydesdorff (2003). “Mapping Global Science Using International Co-authorships: A comparison of 1990 and 2000". En: J. Guohua, R. Rousseau, W. Yishan (Eds.), Proceedings of the 9th International Conference on Scientometrics and Informetrics. Dalian: Dalian University of Technology Press, pp. 330-340. 